$13^{\text {th }}$ International Conference on

AEROSPACE SCIENCES \& AVIATION TECHNOLOGY,

ASAT- 13, May 26 - 28, 2009, E-Mail: asat@mtc.edu.eg

Military Technical College, Kobry Elkobbah, Cairo, Egypt

Tel : +(202) $24025292-24036138$, Fax: +(202) 22621908

\title{
Numerical Investigations of Footing Width Influence on the Surface Footing-Tunnel Interaction Analysis
}

\author{
Nabil M. Nagy*
}

\begin{abstract}
With the increasing in urbanization, tunnels became a necessity for major cities like Cairo and a large number of tunnel projects had to be undertaken worldwide. Tunnels are constructed to resolve transportation and sewerage problems and may pass near or under existing buildings. Conversely, constructing structures on top of existing tunnels could cause stresses increasing within the soil mass, which sequentially increase stresses transferred to and deformation of the tunnel lining. The interaction between the surface footings and underground tunnels depends upon several factors including the relative dimensions of the surface footings and tunnel as well as depth and eccentricity of the proposed tunnel. In this paper, a numerical investigation of a surface footing-soil-tunnel system is performed using the ABAQUS finite element code. To validate the numerical procedure and material constitutive models used in the present work, a comparison with relatively small scale experiments is first performed. The results obtained illustrate that an agreement between numerical and experimental results is reasonable. Further numerical investigations have been carried out for the different factors affecting the system response.
\end{abstract}

Keywords: Footing-tunnel interaction, Numerical modeling, surface footing.

\section{1-Introduction}

Buried structures such as culverts, shelters, pipes and tunnels are vital elements in resolving jamming problems, conveying sewage to treatment plants and protecting public from terrorist attacks and in military usage as well. Due to increasing urbanisation, these structures might pass/be found under or near existing buildings. For example shallow underground structures built in developed areas could result in a significant ground movement if care is not undertaken. Another scenario is when a structure is built above an existing buried structure. In this case the buried structure may suffer from increased pressure, which might cause some distress within the lining and result in excessive deformation. Thus, it is crucial that to improved knowledge about the interaction between surface loads and buried structures.

The theory of bearing capacity of soils is originally developed for a strip footing on homogeneous sand and clay soils [1] and is then extended to reflect many factors including the shape of footing and loading condition (see for example, [2]). The theory of bearing capacity can not be applied if the subsurface soils contain voids or tunnels. Unsupported underground voids could occur naturally due to for example solution cavities. Baus and Wang (1983) [3] studied the settlement behaviour of continuous footings located on a subsurface

\footnotetext{
*Egyptian Armed Forces, nabilmnagy@yahoo.com
} 
soil containing a void. Their study indicated that the settlement of footings is dependent upon the location and size of the underground void. However, if the void exists below a certain depth which is often called the critical depth, the presence of the underground void has a negligible effect on the bearing capacity and associated settlement [3]. This was further elaborated by [4] who concluded that if the void is located within what is called the critical zone, there will be a reduction in the bearing capacity as well as increased settlement. The critical zone then considers both the depth and eccentricity of the void. Practical solutions such as shotcrete or ground supports are implemented to reduce the risk of voids failure. For manmade underground structures, lining and reinforced concrete are used to build sustainable and durable structures. Several investigations have been carried out numerically (see for example, [5] and [6]). [7] investigated circular tunnels installed in elastic ground.

In order to validate not only the material models and corresponding properties but also the analysis procedures; a validation stage is initially conducted by comparing the results of a series of physical tests undertaken by [8] with those obtained from a detailed numerical modeling of the same physical problem. This validation stage has been done successfully previously by [8]. This enabled a calibration of the model and material parameters.

\section{2- Physical Experiments}

\section{1-Experimental set-up and measurements}

A specially designed tank with internal dimensions of $1050 \mathrm{~mm}$ long, $200 \mathrm{~mm}$ wide and 600 $\mathrm{mm}$ deep is used in this investigation. Figure 1 shows a schematic diagram of the tank and loading system. The front wall of the tank is made of $10 \mathrm{~mm}$ thick SECURIT glass in order to allow visual observation and to withstand pressure. The back wall of the tank is made of steel strips with $100 \mathrm{~mm}$ height stiffened with steel angles. In order to change the tunnel dimensions, three strips with different heights are manufactured. The strips are designed in a way so that they can be bolted together in a way to facilitate the variation of tunnel depth and eccentricity between tunnel and footing centres. All strips are insulated with a rubber gasket to ensure a tight fit. A static load is applied using a loading device having 1:10 amplification. Measurements of deformation and strain are carried out at the tunnel crown and both opposite sides (walls). All measurements are taken at the middle of the tunnel lining which is at 100 $\mathrm{mm}$ away from the edge of the tank. Three strain gauges were used to measure the strain in the tunnel lining at the same three locations on the tunnel lining but from outside. Strain gauges are mounted around the tunnel lining before insertion. They are connected with a strain indicator and initial readings are taken. Readings of deformation and strain were taken after each load increment.

\section{2- Testing procedure}

The tank is filled up with the silty sand in $50 \mathrm{~mm}$ layers up to the level of the tunnel bed. Each layer is compacted manually. Then the tunnel lining is inserted from the back wall through the opening in the corresponding strip. The tunnel lining was kept in place using a wooden mould. The filling process is then continued as before till reaching the final predetermined level. Upon completion of tank filling, a second stage of compaction is started by placing a relatively heavy weight plate on top of the sand surface and shaking the whole tank for 75 seconds. After completing the compaction process, the sand surface was carefully levelled off and any extra sand is carefully removed. The footing was then placed on the 
centre of the tank and the loading device is attached to the footing. The load is applied in steps and continued until soil or tunnel failure occurs.

\section{3- Numerical Modeling}

In this paper, the non-linear finite element programme ABAQUS/Standard is used to undertake a two-dimensional (2D) plain strain analysis of a miniature of a horseshoe-shaped tunnel that is embedded in sand same as the experimental setup. Finite Element Method is employed for investigating the mutual effects between surface loads and tunnels. A numerical model is developed so that the considered problem can be investigated. An elaborated description of the models assigned for soil mass, tunnel structure and interaction between tunnel and surrounding soil is presented in this section. The soil material is modelled using the inelastic Mohr-Coulomb material model. The tunnel lining and footing are modelled as linear elastic materials. The geometrical shape and dimensions of the problem as shown in Figure 2.

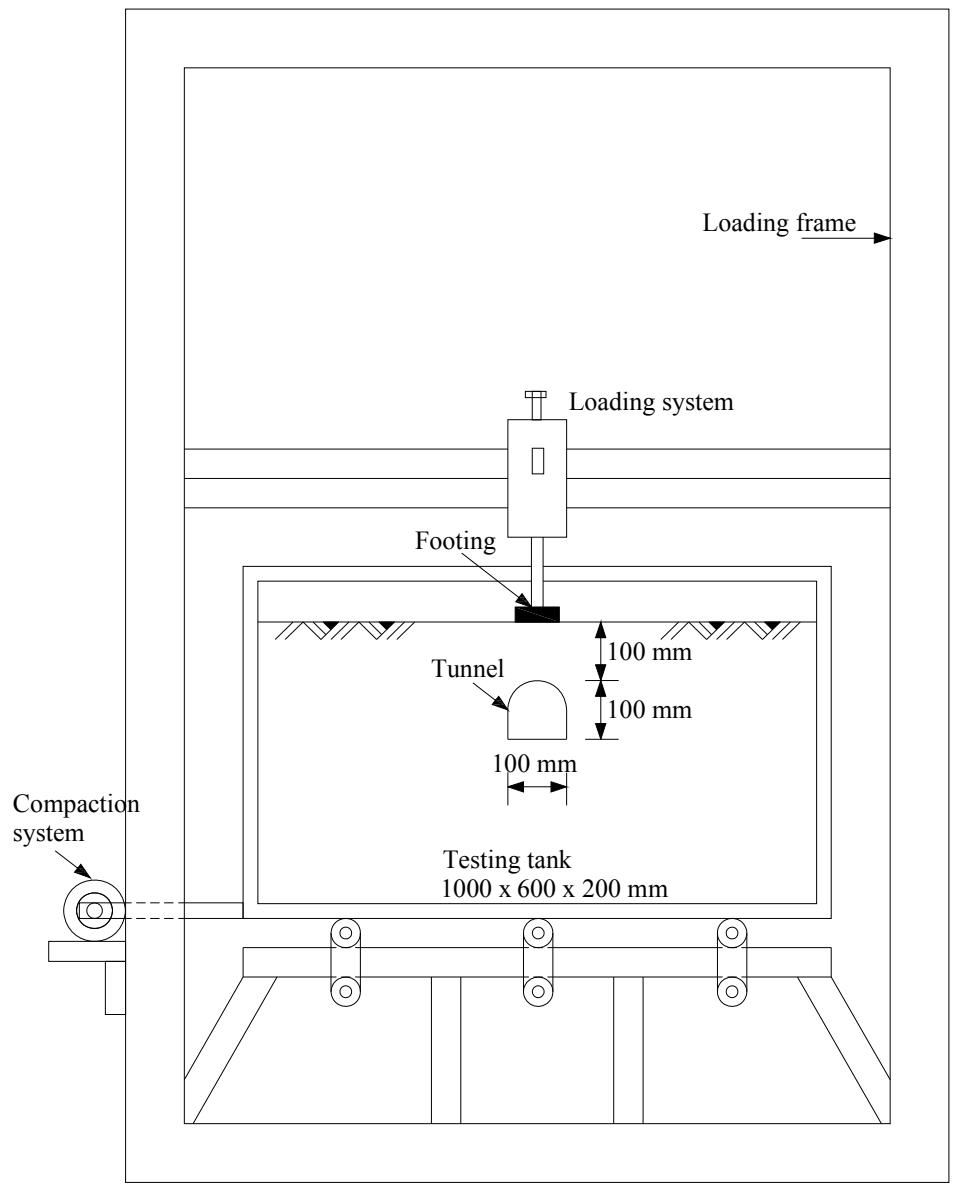

Figure 1: Schematic diagram of the experimental set-up 


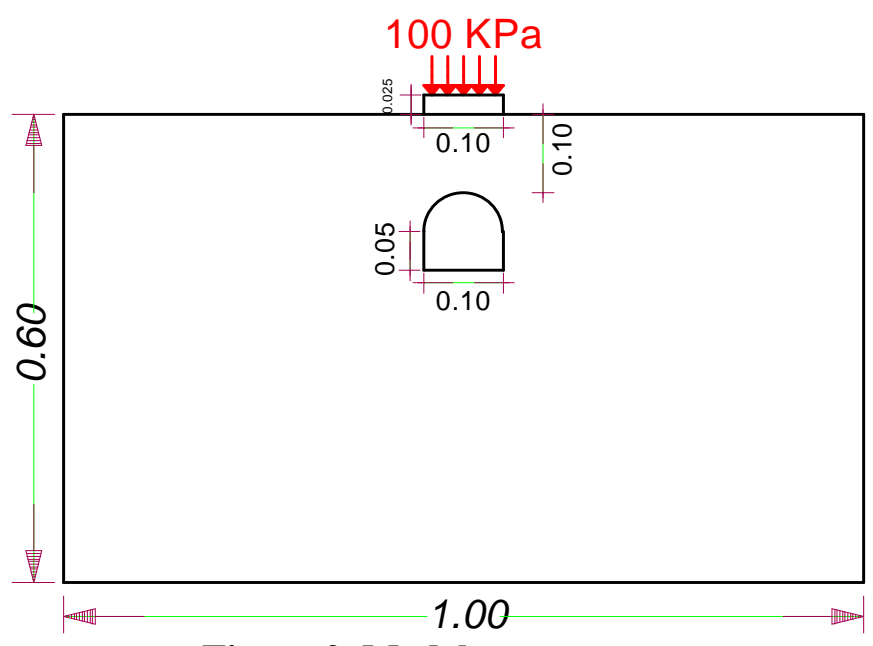

Figure 2 Model parameters

\section{1- Material Models}

The soil is considered as a dense silty sand material whose behaviour is modelled by an elasto-plastic Mohr-Coulomb material model. This model uses the classical Mohr-Coulomb yield criterion including a straight line in the meridional plane and a six-sided polygon in the deviatoric plane. However, the ABAQUS Mohr-Coulomb model has a completely smooth flow potential instead of the classical hexagonal pyramid. The flow potential is a hyperbola in the meridional plane, and uses a smooth deviatoric section proposed by [9]. The values of Young's modulus and possion's ratio are assumed to be $7 \mathrm{MPa}$ and 0.3 respectively. Since the tunnel lining is made up of Galvanized steel, its behavior can be described as an elastic material. The galvanised steel lining has a density of $7800 \mathrm{Kg} / \mathrm{m}^{3}$, Young's modulus of 200 $\mathrm{GPa}$, Poisson's ratio $(v)=0.3$ and rigidity of $2.55 \mathrm{X} 10^{-5} \mathrm{KNm}^{2}$.

\section{2- Soil-tunnel Interaction}

A precise simulation of the soil structure interface is vital for a successful modelling of the structural response. The soil-tunnel interaction is modelled using "Contact pair" models. A pure master-slave relationship is used to model contact between two surfaces [10]. In this case the outer surface of the tunnel structure is modelled as "Master Surface" and the surrounding soil surface is modelled as "Slave Surface". In the ABAQUS calculations, a "finite sliding" formulation is used. Finite sliding allows for any arbitrary motion of the surfaces such as separation, sliding, rebound and rotation of the surfaces in contact, which should be needed in the analysis of the structure [11].

\section{3- Model discretization}

A 3-node bilinear plane strain quadrilateral, reduced integration are used to model the sand medium with dimensions of $600 \mathrm{~mm}$ deep and $1000 \mathrm{~mm}$ length. In addition, a 4-node bilinear plane strain quadrilateral, reduced integration elements are used to model the tunnel lining. The footing is modelled as steel, rigid and perfectly bonded to the soil surface. Horizontal displacement boundary conditions are applied along the vertical edges by restraining the displacement in the horizontal direction and vertical displacement boundary conditions are applied along the base edge by restraining the displacement in the vertical direction. The 
overall geometric model is divided into three different regions representing the soil, tunnel and the rigid surface footing. A convergence study involving mesh refinement, appropriate selection of element types is initially performed; hence the optimum meshes are identified for the soil, tunnel and the rigid surface footing, as shown in Figure 3.

It should be noted that automatic meshing is employed and therefore there might be slight difference in the elements around the central vertical axis. However, denser mesh is constructed in areas in which stresses are concentrated. An incremental external uniform pressure is applied on the footing. The pressure is varied between 0 to $500 \mathrm{kPa}$ on $100 \mathrm{kPa}$ increments. The tunnel lining is modelled as galvanized steel with constant thickness $(t)$ of $0.35 \mathrm{~mm}$. Both the internal height of the tunnel $(h)$ and tunnel width are kept at $100 \mathrm{~mm}$. The tunnel is buried in the sand at a depth $(d)$ of $100 \mathrm{~mm}$ and centred below the surface footing as shown in Figure 3. The entire footing-soil-tunnel system is represented by an assemblage of a finite number of 3 ad 4-node bilinear plane strain quadrilateral, reduced integration elements.

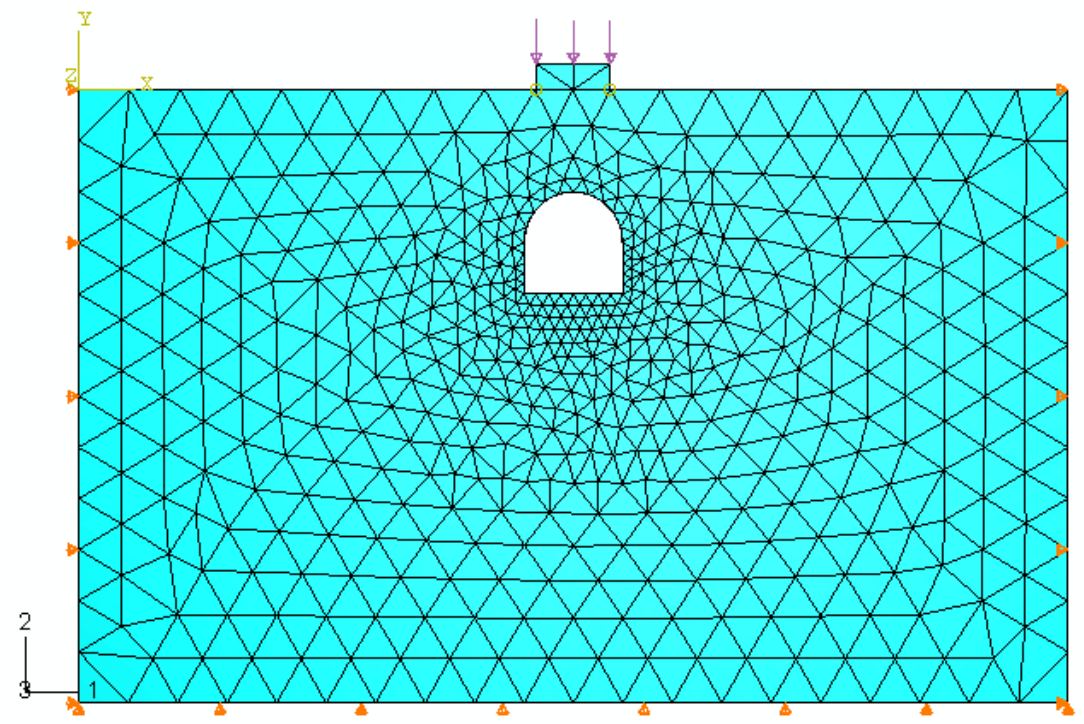

Figure 3: Finite element Model discretization

\section{4- Results and discussion}

Many numerical models were executed to investigate the interaction between surface loading and buried tunnels. In this paper only the results for concentric tunnel that is located $100 \mathrm{~mm}$ beneath the surface footing are presented and discussed in case of varying footing width in order to analyze the influence of footing width on the system response.

\section{1- Footing settlement}

Three numerical models for different footing widths of 50,75 and $100 \mathrm{~mm}$ are investigated in this study. The tunnel width is $100 \mathrm{~mm}$. The tunnel deformation is measured at three different positions namely crown, side/wall 1 and side/wall 2 . Since the footing is concentric with the tunnel, deformation of side 1 and side 2 are expected to be the same. Thus, only deformation of one side is presented. 
Figure 4 shows the results of footing settlement as a function of the footing pressure for different footing widths. The results demonstrate clearly that for the same tunnel width and depth, any increase in the footing width causes an increase in the footing settlement. The results demonstrate that the measured settlement for $75 \mathrm{~mm}$ and $100 \mathrm{~mm}$ footings is almost same up to a bearing pressure of $200 \mathrm{kPa}$. Then noticeable difference is clear for footing pressures above $200 \mathrm{kPa}$. Figure 4 shows that for the $50 \mathrm{~mm}$ footing, settlement increases slightly with footing pressure. Only a $0.5 \mathrm{~mm}$ settlement is recorded at a pressure of $200 \mathrm{kPa}$, $2.8 \mathrm{~mm}$ at $500 \mathrm{kPa}$ and failure occurs at $520 \mathrm{kPa}$. For larger footings, the footings experienced $\sim 1.0 \mathrm{~mm}$ settlement at a pressure of $140 \mathrm{kPa}$. Then sharp increase in the rate of settlement occurred with any further increase in the footing pressure. The ultimate pressure is $310 \mathrm{kPa}$ and $260 \mathrm{kPa}$ for footing width of $75 \mathrm{~mm}$ and $100 \mathrm{~mm}$ respectively.

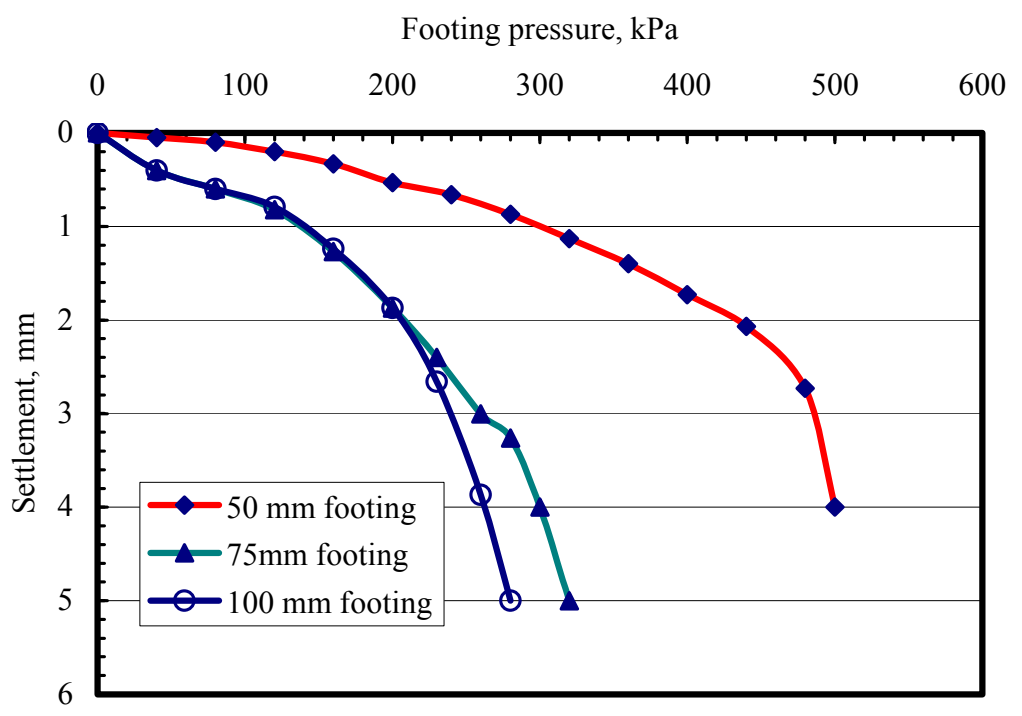

Figure 4: Settlement of surface footing against footing pressure

\section{2- Tunnel Deformation}

The influence of footing width on crown deflection for the same tunnel width and depth is shown in Figure 5. It can be seen that crown deflection increases as the footing pressure increases. For $75 \mathrm{~mm}$ and $100 \mathrm{~mm}$ footings less crown deflection is recorded for $100 \mathrm{~mm}$ footing up to a footing pressure of $200 \mathrm{kPa}$. The slightly smaller crown deflection for the largest footing at low footing pressure could be due to a stabilization effect of the pressure transferred to the bottom of the tunnel arch. This transferred pressure helps in reducing the deflection of the crown. Generally, crown deflection follows qualitatively the same trend as the footing settlement. This indicates that the footing width would cause an increase in the load transfer to the tunnel lining which in turn causes an increase in the crown deflection. For the smallest footing, failure of tunnel lining was not observed. However, the footing failure was due primarily to the soil cover underneath the footing and above the tunnel.

The results of footing settlement and deflection of tunnel crown show that stresses are concentrated in a zone that is directly under the surface footing. This zone is called the influenced zone and is strongly related to the footing width. Thus for smaller footings the bearing pressure settlement relation would be controlled mainly by the characteristics of the direct soil under the footing with minimal interaction with tunnel since the tunnel depth is 
four times the footing width. As the footing width increases, stress is concentrated in a zone that is directly above the tunnel crown. This would increase the crown deformation. However, with further enlargement of the influenced zone as a result of relatively large footings, stresses are supporting the tunnel sides which would result in a decrease in the measured tunnel crown deflection. In this case, there is a mutual influence on the behaviour of both footing and tunnel. With further increase in footing width, more stresses are concentrated on the tunnel arch leading to large settlement as well as reduced ultimate bearing pressure.

Results of footing settlement and deformation of tunnel obtained numerically are presented in Figures 6. It can be seen that footing settlement increases with the increase in the applied footing pressure with no definite failure for the footing-tunnel system. Settlement reaches the value of $18.5 \mathrm{~mm}$ when the applied footing pressure is $500 \mathrm{kPa}$. Comparing data for the footing settlement obtained numerically with actual records of settlement from physical testing demonstrates that numerical simulation does not capture the failure of the footingtunnel system at $280 \mathrm{kPa}$ nor its results are close to the measured. Interesting results for the tunnel base deformation show that the corner of the tunnel always suffers a settlement whereas the deformation of the tunnel base centre is upward.

Deformation of the ground surface is also obtained numerically at various applied footing pressure and shown in Figure 7. The data presented in Figure 7 depict that most of the deformation occurs underneath the footing which in this investigation is located $100 \mathrm{~mm}$ above and concentrically with the tunnel. The results clearly show that no heave would be experienced which is contradictory to the observation in the laboratory. This could be due to limited capabilities of the model assigned to simulate the soil behaviour. Results of the vertical stress distribution in the soil medium are presented in Figure 8 for an applied footing pressure of $500 \mathrm{kPa}$. These results demonstrate clearly that for the current problem, arching is working on resulting in the transfer of portion of the developed vertical stress to the sides of the tunnel supporting the tunnel arch. These results explain the measured crown deflection presented in Figure 5. Most of the stresses are concentrated on the tunnel arch. Stresses are reduced towards the tunnel base. Higher stresses applied on the sides of tunnel would be offering a stabilising effect reducing the deformation of tunnel crown.

To monitor the footing width influence on the surface footing-tunnel system, Figures 9- 17 show the horizontal and vertical displacement and deformed shape contours for the system under surface footing pressure of $500 \mathrm{Kpa}$. Further investigations are on the go to determine more appropriate and meaningful soil parameters as well as to the use of more sophisticated soil models such as Draker-Prager's model and cap model.

\section{5- Conclusions}

In this paper an investigation is carried out into the interaction between footing and shallow tunnel buried in silty sand. A number of physical tests are conducted in which deformation of footing and tunnel is measured. In addition preliminary numerical simulations are undertaken using numerical code "ABAQUS" in order to validate the numerical model. The validated model has been used to undertake more investigations on the footing-tunnel system. Soil and Galvanised steel are modeled as an elastic-plastic and elastic materials respectively. The following are some of the main conclusions that can be made out of the investigation: 
For small footings, it is most likely that the behaviour of footings will be controlled by the soil behaviour under the footings rather than by the tunnel. For medium and large footings, it is likely that there will be a mutual interaction between the surface footings and buried tunnels resulting in a relatively increased deformation of both structures. For large footings, stresses might develop on the sides of the tunnel resulting in a stabilising pressure leading to a slight reduction on the tunnel and footing settlements. However, as the applied footing pressure increases, large settlement occurs. The results of the numerical simulations show that footing settlement increases with the applied footing pressure with no obvious failure. In addition the numerically obtained results for stress distribution in the soil clearly explain the behaviour of the footing and tunnel system. Numerically obtained deformations are much greater than those obtained experimentally, raising question marks concerning the model assigned for capturing the behaviour of the soil and steel materials. Further investigations are underway to determine accurate values for the soil parameters and to use a more sophisticated model for the soil.

\section{6- References}

[1] Terzaghi, K. (1943). Theoretical Soil Mechanics. John Wiley \& Sons, New York.

[2] Meyerhof, G.G. (1963). Some recent research on the bearing capacity of foundations.Canadian Geotechnical Journal, Vol 1(1).

[3] Baus, R.L. and Wang, M.C. (1983). Bearing Capacity of strip footing above void Journal of Soil Mechanics and Foundation Engineering, ASCE, Vol 109(1) pp 1-13.

[4] Wood, L.A. and Larnach, W.J. (1984). The behaviour of footings located above voids. Proceedings of the Eleventh International Conference on Soil Mechanics and Foundation Engineering, Vol. 4, pp 2273-2276.

[5] Dessouki, A.K. and Monforton, G.R. (1986). Effect of soil failure on soil-steel structure. Journal of Geotechnical Engineering, ASCE, Vol112 (5), pp 522-536.

[6] Duddeck, H. (1987). Generl Approaches to the design of underground openings. VI Australian Tunnelling Conference, Melbourne, pp 159-172.

[7] Muir-Wood, A.M. (1975). The behaviour of circular tunnel in elastic ground. Journal of Geotechnique, Vol. 25 (1), pp 115-127.

[8] Mostafa Mohamed and Nabil Nagy (2008). Numerical and experimental investigations into the interaction between surface footings and shallow tunnels. 7 th International Conference on Civil \& Architecture Engineering. Military Technical College. Cairo, Egypt.

[9] ABAQUS Theory Manual (2005), Version 6.5. Published by Hibbitt, Karlsson and Sorensen Inc. USA.

[10] ABAQUS Analysis Manual (2005), ABAQUS Analysis User's Manual, Version 6.5, Published by Hibbitt, Karlsson and Sorensen Inc. USA.

[11] Helwany, S. (2007), Applied soil mechanics with ABAQUS`applications. Hoboken, New Jersey: John Wiley \& Sons, INC. 


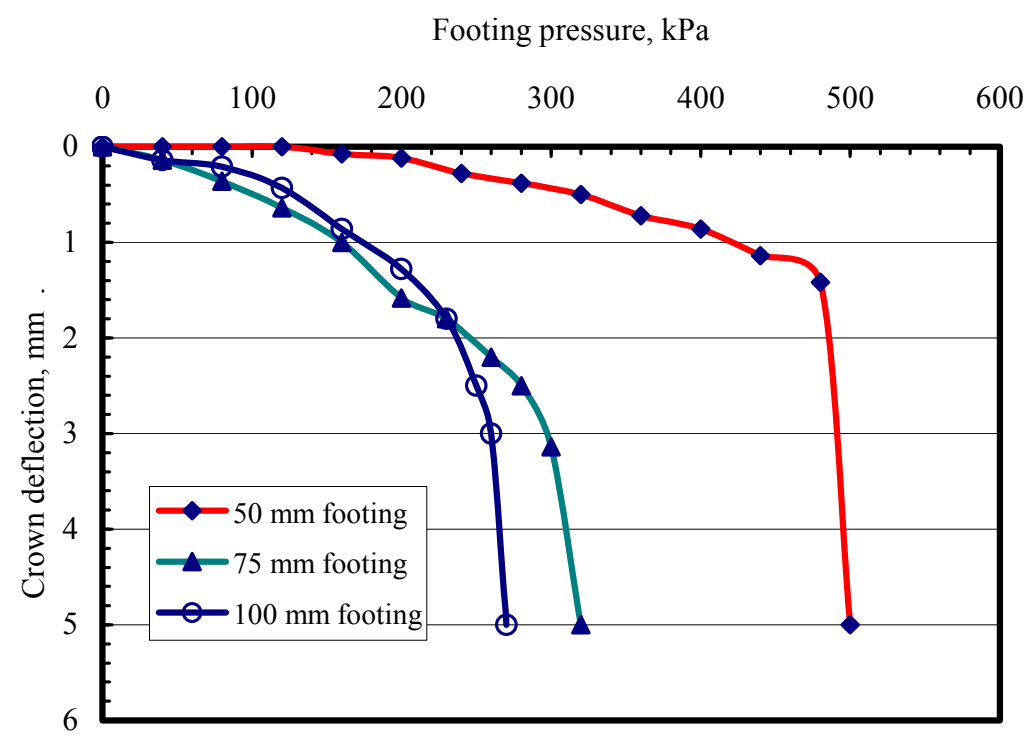

Figure 5: Crown deflection against the footing pressure for different footing widths

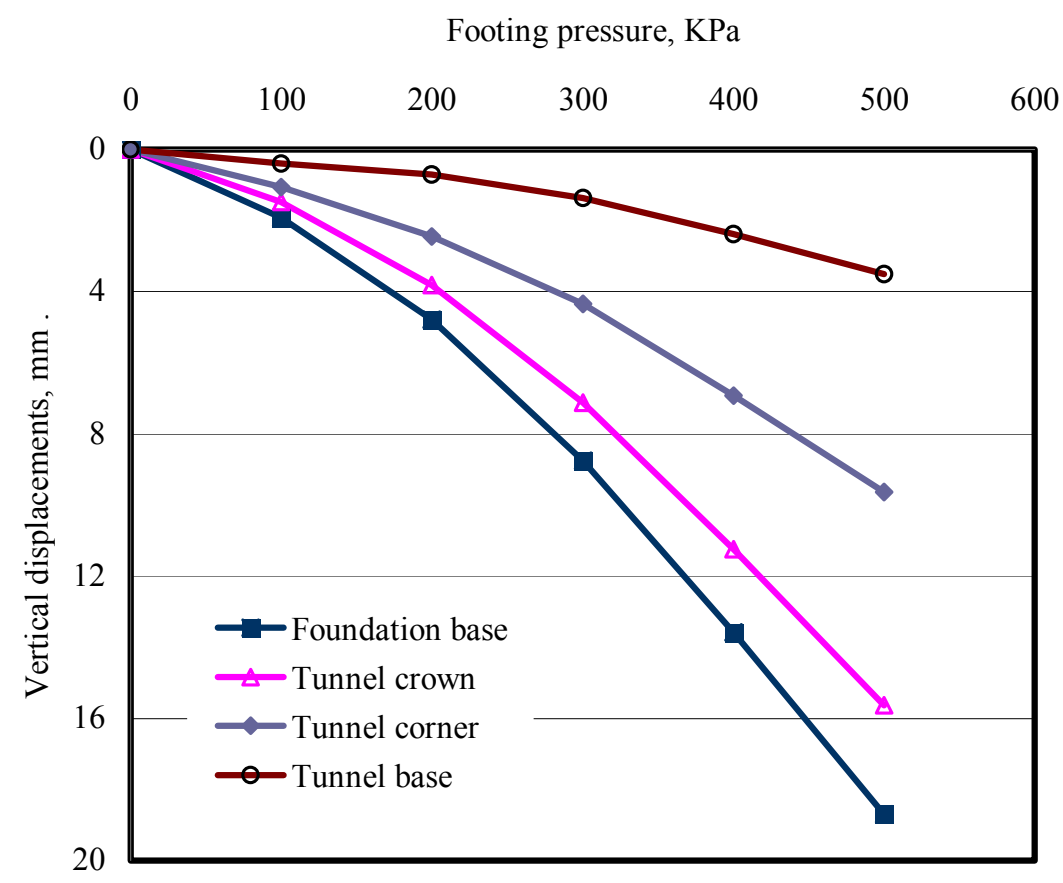

Figure 6: Numerically obtained results for settlement 


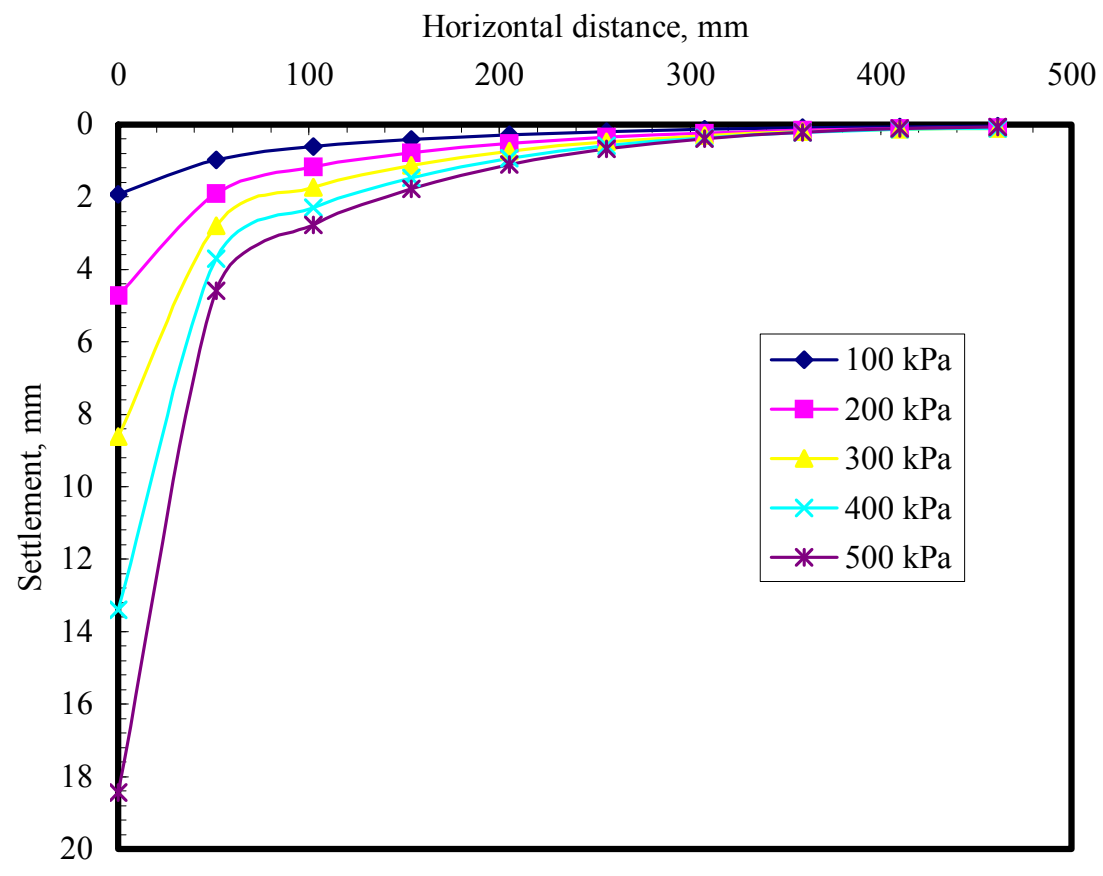

Figure 7: Numerical results for ground settlement

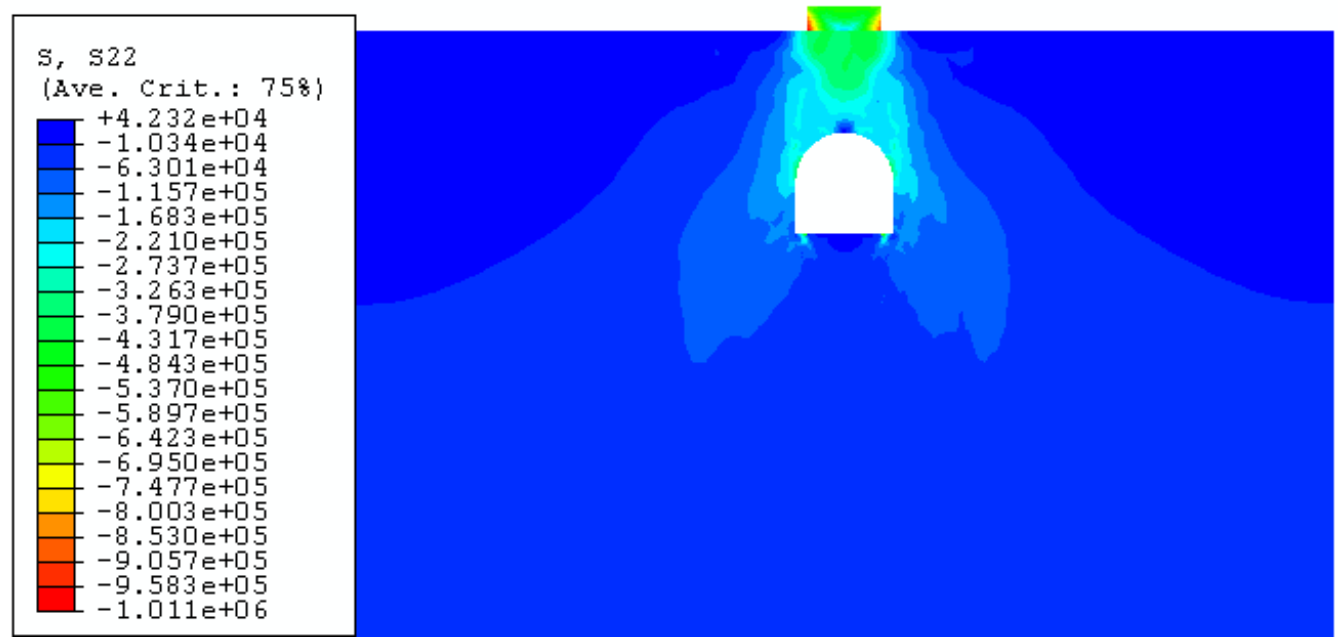

Figure 8: Stress distribution for an applied footing pressure of $500 \mathrm{kPa}$ 


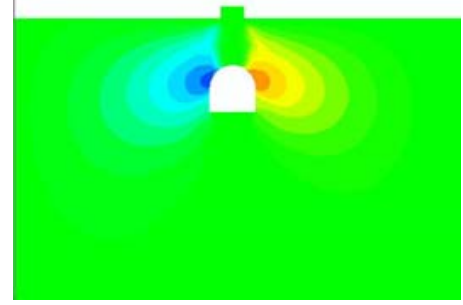

Figure 9: horizontal displacement of the tunnel-footing system for $50 \mathrm{~mm}$ footing width

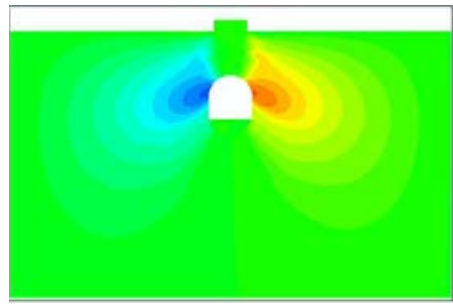

Figure 12: Horizontal displacement of the tunnel-footing system for $75 \mathrm{~mm}$ footing width

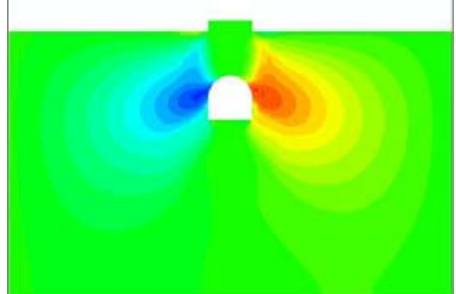

Figure 15: Horizontal displacement of the tunnel-footing system for $100 \mathrm{~mm}$ footing width

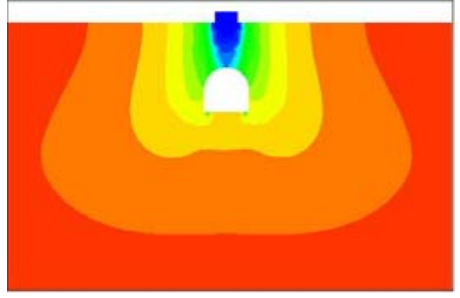

Figure 10: vertical displacement of the tunnel-footing system for $50 \mathrm{~mm}$ footing width

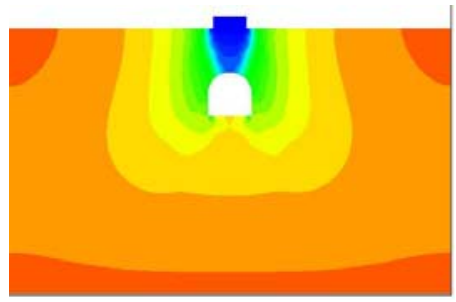

Figure 13: Vertical displacemen of the tunnel-footing system for $75 \mathrm{~mm}$ footing width

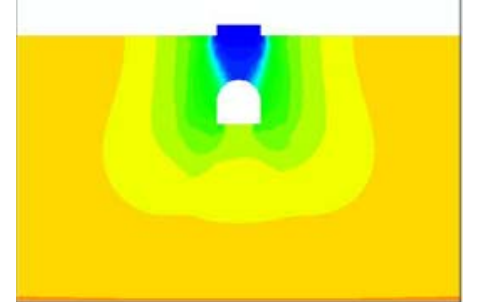

Figure 16: Dertical displacement of the tunnel-footing system for $100 \mathrm{~mm}$ footing width

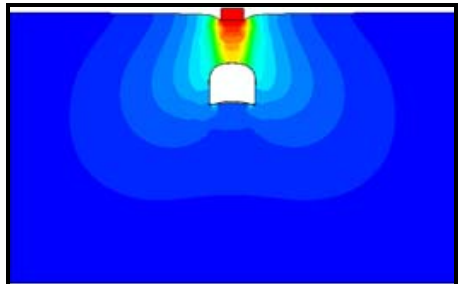

Figure 11: deformed shape of the tunnel-footing system for $50 \mathrm{~mm}$ footing width

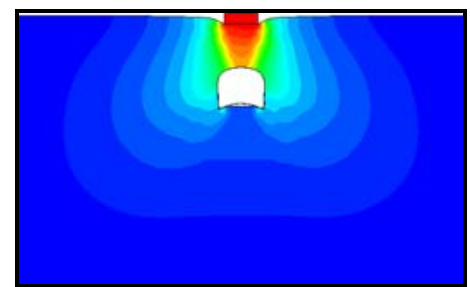

Figure 14: Deformed shape of the tunnel-footing system for $75 \mathrm{~mm}$ footing width

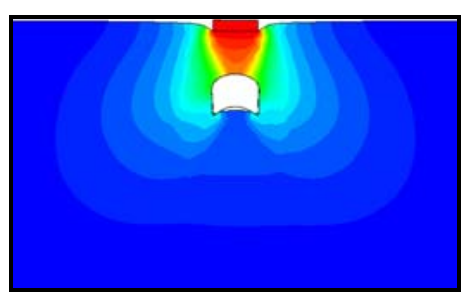

Figure 17: Deformed shape of the tunnel-footing system for $100 \mathrm{~mm}$ footing width 